Research Paper

\title{
Ligands Binding and Molecular Simulation: the Potential Investigation of a Biosensor Based on an Insect Odorant Binding Protein
}

\author{
Xin Yi, Yanbo Zhang, Peidan Wang, Jiangwei Qi, Meiying Hu, Guohua Zhong ${ }^{\bowtie}$ \\ Laboratory of Insect Toxicology, Key Laboratory of Pesticide and Chemical Biology, Ministry of Education, South China Agricultural Uni- \\ versity, Guangzhou, People's Republic of China.
}

\begin{abstract}
$\square$ Corresponding author: Prof GuoHua Zhong. Key Laboratory of Pesticide and Chemical Biology, Ministry of Education, PR China, College of Natural Resources and Environment, South China Agricultural University, Guangzhou 510642, China. E-mail: guohuazhong@scau.edu.cn. Tel: +86-20-85280308; Fax: +86-20-85280292.
\end{abstract}

(C) Ivyspring International Publisher. This is an open-access article distributed under the terms of the Creative Commons License (http://creativecommons.org/ licenses/by-nc-nd/3.0/). Reproduction is permitted for personal, noncommercial use, provided that the article is in whole, unmodified, and properly cited.

Received: 2014.06.11; Accepted: 2014.11.05; Published: 2015.01.01

\begin{abstract}
Based on mimicking biological olfaction, biosensors have been applied for the detection of various ligands in complex environment, which could represent one of the most promising research fields. In this study, the basic characters of one insect odorant binding protein (OBP) as a biosensor were explored. To explore the molecular recognition process, the tertiary structure of the protein was modeled and the protein-ligand interactions with 1,536,550 chemicals were investigated by the molecular docking. The availability of large amount of recombinant SlitOBPI overcame the difficulty to obtain biological sensing material. After obtained the purified recombinant protein, the result of fluorescence binding assays proved the candidate protein has good affinities with the majority of the tested chemicals. With the aid of simulation docking, the key conserved amino acids within the binding site were identified and then mutated to alanine. After mutation, the protein-ligand binding characteristics were recorded, and the competitive binding assays were carried out to provide experimental verification. The detailed information on its structure and affinities investigated in this study could allow the design of specific mutants with desired characteristics, which provides a solid base for tailoring OBP for biosensor and provides a role model for screening the other elements in olfactory system for different applications.
\end{abstract}

Key words: Odorant binding protein, Biosensors, Molecular simulation, Fluorescence binding, Site-directed mutagenesis.

\section{Introduction}

The demand for versatile technologies that can be used as sensors for the detection of volatile chemicals has rapidly increased in the last decade for various applications, including homeland security [1], environmental pollution control, health and wellness [2]. These sensors possess features including precision, reversibility, selectivity, low cost, versatile sensing and compactness [3]. Biological olfactory system are highly sensitive and have broad detection spectra [4], and thus represent excellent candidates for biomimetic sensors [5-8]. Indeed, the sensor based on biological olfactory system only requires a protein with reversible binding capability [9]. However, intensive efforts have been taken to determine the pharmacological profile and ligands specificities of individual olfactory receptors, until now, only a few studies focus on the odorant binding proteins (OBPs). OBPs are important components of chemosensory system $[10,11,12]$, and have attracted increasing attention in recent years due to their great potential as 
biosensing elements [13-15]. Indeed the increasing understanding of biochemical, physiological and behavioral aspects of ligands sensation could promote the development of biosensor $[16,17]$. The exceptional stability of OBPs to thermal denaturation and proteolytic degradation also made these proteins ideal for use in sensing devices [18, 19]. However, there are only limited research papers describing the basic characteristics of OBPs as biosensors.

In fact, in insects, OBPs have two major roles: detection and delivery of chemical stimuli. They are associated to olfaction, chemoreception and highly concentrated in the lymph of chemosensilla in insects [20]. Insect OBPs have a highly conserved folding pattern that is completely different from their counterparts in vertebrates [19]. Particularly, the accurate detection of surrounding environment is crucial for insects to survive, thus, many insects have developed intricate, sensitive and specific tuned olfactory systems to detect the differentiate compounds at high resolution [21, 22]. Moreover, due to the high complexity of the chemical language used by Spodoptera litura to communicate, investigation of the olfactory system in OBPs of this insect is of particular interest and insect OBPs are perhaps best known in the order Lepidoptera [23]. Each OBP could recognize a range of semiochemicals with vastly different molecular shapes and sizes, functional groups, charge, hydrophobicity and even concentration [24]. A better understanding of the selectivity of these OBPs towards different semiochemicals could facilitate the design of sensor for specific chemicals.

Previously, Spodoptera litura OBP1 was cloned and the expression was investigated by our team. The OBP1 showed distinct conservation in the evolution and this specific protein showed $78.1 \%, 87.6 \%$ and $79.9 \%$ identity to the OBP1 of the Helicoverpa assulta, Heliothis virescens and Helicoverpa armigera, respectively [25]. In addition, among the 16 known OBPs in S. litura, OBP1 was found to be up-regulated in response to several chemicals (Unpublished data), which indicated it equipped diverse sensing abilities. Together with previously established characteristics, the OBP1 from $S$. litura is a promising candidate. In this study, a three dimensional (3D) model of OBP1 was constructed by homologous modeling, and the plausible binding modes between the model protein and 1,536,550 molecules were then inferred by docking simulation to determine its affinities to different molecules. Large amount of chemicals were screened in this study to demonstrate the binding activities of SlitOBP1 as a multisensor, and using the identified binding characters, the SlitOBP1 could be specifically modified to use as biosensors in monitoring certain ligands. This study demonstrated insect OBPs have broad ligand sensing capabilities and can be modified to be used as specific chemical biosensors to provide foundation for fabricating biosensor as well as other biotechnological applications and to provide a potent method for building multisensor device for ligands discriminations.

\section{Materials and Methods}

\section{Insect}

An artificial diet was applied for rearing S. litura (F.) [26] at $25 \pm 1{ }^{\circ} \mathrm{C}$ in a $14: 10$ light : dark photoperiod and $60-70 \%$ relative humidity (RH). The new emerged adults were transferred to Chinese cabbage [Brassica campestris L.ssp. Chinensis (L.)] and raised at $25{ }^{\circ} \mathrm{C}$ and $60-70 \% \mathrm{RH}$. Three and four days after emergence, the adult moths were used for extraction of RNA.

\section{Chemicals}

The Available Chemicals Directory (ACD, Asinex Ltd Moscow, Russia), comprising 1,579,000 compounds, were used to investigate the binding affinities of SlitOBP1 in simulation docking. 21 ligands with the highest purity were purchased from Sigma (St Louis, USA) and stored according to the manufacturer's instructions to investigate the ligand-binding activities of SlitOBP1.

\section{Sample preparation and RNA isolation}

The total RNA from S.litura was extracted by the E.Z.N.A.TM total RNA isolation system kit (Omega, USA) according to the manufacturer's instructions. The M-MLV reverse transcriptase (TaKaRa, China) and oligo(dT) ${ }_{18}$ was used to transcribe the isolated RNA to first-strand cDNA at $42{ }^{\circ} \mathrm{C}$ for $60 \mathrm{~min}$. After heating at $95{ }^{\circ} \mathrm{C}$ for $5 \mathrm{~min}$, the reaction was terminated, and then stored at $-20{ }^{\circ} \mathrm{C}$.

\section{Alignment and homology modeling}

A blast search of the full length of cloned SlitOBP1 (accession number EF159978) was conducted against the current Protein Data Bank (PDB; http:/ / www.rcsb.org) to find structural template. The initial model was constructed by DS MODELER [27] in Discovery Studio 2.1 (Accelrys Software Inc.). To refine the initial homology model, the CHARMm force field was employed and three energy minimization procedures were processed. After performing 1000 steps of steepest descend (SD) and 2000 steps of conjugate gradient (CG) minimization, a molecular dynamic (MD) simulation was carried out to examine the quality of the model structure. First, minimization was carried out while all of the hydrogen atoms were relaxed and the other atoms were fixed. Then, the side-chains were energy-minimized while the main 
chain was restrained. Finally, minimization was performed while all the atoms were relaxed. The non-bond cutoff distance of $7 \AA$ was used, and the long-range electrostatic interaction was calculated using the spherical cutoff method [28]. The final 3D model was evaluated by PDF total energy, verify score [29] and Ramachardran plot [30].

\section{Molecular docking}

The compounds in the Available Chemicals Directory (ACD) database were screened by the Lipinski's rule of five in the ISIS BASE. The 'rule of five' stated that: poor absorption or permeation are more likely when: there are more than $5 \mathrm{H}$-bond donors (expressed as the sum of $\mathrm{OHs}$ and $\mathrm{NHs}$ ); The MWT is over 500; The $\log \mathrm{P}$ is over 5 (or MLogP is over 4.15); There are more than $10 \mathrm{H}$-bond acceptors (expressed as the sum of Ns and Os). The active site pockets of the receptor were found automatically by the Binding site in Discovery Studio 2.1. Binding site uses a CHARMm-based molecular dynamics scheme to seek for the optimal binding sites for docking [31]. Based on the analysis of the geometry shape of the surface of the protein, the cavity which could bind to the substrate was identified, and the potential binding sites were predicted. Then, the optimal binding site was chosen based on the shape and location of the cavity, the location of the residue and the conserved acid amino. A site sphere radius was set to assign the entire binding pocket. Other parameters were set as default. The docking program Ligandfit and CDOCKER were used to perform the automated molecular simulation. The 'Number of Monte Carlo Trials' and 'Energy Grid' in the Ligandfit were used to first round screening. Electrostatic energy was excluded in the calculation of the ligand internal energy. Other parameters were set as default, the Extension from site was set as 3.0, the Nonbonded cutoff distance was set as 10.0, the Maximum internal energy was set as 10000, the RMS threshold for ligand/site match was set as 2.0, the rigid body SD iterations was set as 10, the Rigid Body BFGS iterations was set as 20, and four scoring function were applied. Subsequently, the CDOCKER was used for second screening. The Top hits was set as 10, the Random Conformations was set as 10 . The top compounds were ranked by the corresponding values of -CDOCKER energy, -CDOCKER interaction energy, -PLP1 and Ligscore1, and all the values were preserved to find the most probable binding mode. During the analysis of the screened chemicals, the two scoring functions, PLP1 and LigScore1 appeared to be more precisely than other two functions. After evaluating the scores, conformation and detailed binding mode by these two functions, and combining with other two, 21 chemi- cals were selected for the subsequent analysis.

\section{Expression of Recombinant and Purification for SlitOBP 1}

By using gene-specific primers with the restriction enzyme sites (F-5'-CGCGAATTCATGTTG CTGTTGTTGCGCGC-3', R-5'-CCGCTCGAGTCAGC GCGCCTCAGCCTCCA-3'), the sequence encoding SlitOBP1 was amplified with ExTaq DNA polymerase (TaKaRa, Japan). By T4 DNA ligase (Takara, China), the PCR product was connected to pET28a (Invitrogen, US) and then transformed to BL21 (DE3) competent cells (Takara, China). After the identification by PCR, the positive clone was inoculated in liquid LB overnight at $37{ }^{\circ} \mathrm{C}$. When its $\mathrm{OD}_{600}$ reached 0.4-0.6, 1 $\mathrm{mM}$ Isopropyl-D-thiogalactoside (IPTG) was added to incubate for another $12 \mathrm{~h}$ at $28{ }^{\circ} \mathrm{C}$. The total protein was purified from the supernatant by affinity chromatography using HisTrap columns (GE Healthcare). After dialysis in Tris- $\mathrm{HCl}(\mathrm{pH}=7.4)$ overnight, the Bovine Enterokinase was used to remove the His-tag. The purified protein was collected and examined by 12\% SDS-PAGE and Western blot. Bradford method was used to determine protein concentration [32].

\section{Fluorescence-Based Ligand Binding Assays}

The F-4500 FL Fluorescence Spectrophotometer (HITACHI) was used to record fluorescence spectra in a $1 \mathrm{~cm}$ light path quartz cuvette at $23{ }^{\circ} \mathrm{C}$. The slit width of $5 \mathrm{~nm}$ was used for excitation and emission. The final 21 ligands of different structure and rational binding modes appeared in four scoring functions, and available to purchase, were selected to be the candidates in the competitive binding assay. Chromatographic purity grade methanol was used to dissolve the fluorescent probe $\mathrm{N}$-phenyl-1-naphthylamine (1-NPN) and all ligands used in competition experiments. By exciting the fluorescence of $2 \mu \mathrm{M}$ 1-NPN in $50 \mathrm{mM}$ Tris- $\mathrm{HCl}$ at $337 \mathrm{~nm}$, the affinity of 1-NPN to SlitOBP1 was measured and recorded the emission spectra between $350 \mathrm{~nm}$ and $480 \mathrm{~nm}$. In presence of SlitOBP1 at $2 \mu \mathrm{M}$ and 1-NPN at $2 \mu \mathrm{M}$, the affinities of the selected 21 chemicals were measured by competitive binding assays by adding ligands from 0 to $20 \mu \mathrm{M}$. All values reported were obtained from three independent measurements.

To calculate the binding constants, the corresponding to the maximum fluorescence emission was plotted against the ligand concentrations. The amount of bound ligand was measured using the values of fluorescence intensity with a stoichiometry of 1:1 protein: ligand. The curves were linearized by Scatchard plots. The dissociation constants of the competitors were calculated using the corresponding $\mathrm{IC}_{50}$ values according to the equation: $\mathrm{Ki}=\left[\mathrm{IC}_{50}\right] /(1+$ 
$\left.[1-\mathrm{NPN}] / \mathrm{K}_{1-\mathrm{NPN}}\right)$, where $[1-\mathrm{NPN}]$ is the free concentration of 1-NPN and $\mathrm{K}_{1-\mathrm{NPN}}$ is the dissociation constant of the complex protein/1-NPN [33].

\section{Simulation of site-direct mutagenesis}

All the residues which were within $4.0 \AA$ distances around the molecule in the molecular docking were represented as sites of binding, and the binding free energy between the active site and ligand was calculated by the Calculate Interaction Energy program. When the absolute values of Van der Waals forces interaction energy and Electrostatic interaction energy with ligands were above $2.5 \mathrm{kcal} / \mathrm{mol}$ [34], or formed hydrogen bond or conjugation effect with the ligands, the amino acids were considered as key residues and were selected for mutagenesis study. These key residues were mutated to alanine, respectively. The structural models of the mutants were re-constructed by Discovery Studio as described previously. The binding activities were measured by the simulation docking as previously described. The downrange of the scores in docking were calculated by the formula:

The downrange score after mutation $(\%)=($ The score before mutant- The score after mutant) / (The score before mutant) $\times 100$

Molecular dynamics was applied to evaluate the stability of the binding. The model was used the TIP3P force filed [35]. $\mathrm{Na}^{+}$and $\mathrm{Cl}^{-}$were added to regulate the value of $\mathrm{PH}$. The Energy minimization for optimization of residue geometry was applied until the gradient tolerance was satisfied.

\section{Expression of mutants}

The SlitOBP1 protein was mutated to yield the mutants SlitOBP1 Trp37 (tryptophan to alanine), SlitOBP1 Phe12 (phenylalanine to alanine), SlitOBP1 Ile52 (isoleucine to alanine), SlitOBP1 Thr9 (threonine to alanine), SlitOBP1 Glu98 (glutamic to alanine) by using the quickchange site-directed mutagenesis kit (Agilent) as the recommended procedure. The correct insertion of mutations was verified by DNA sequencing. Expressions of the proteins were carried fout as described previously. SDS-PAGE was used to monitor protein expression and purification. The binding activities were also investigated by the competitive binding assays as previously described.

\section{Results}

\section{Molecular modeling}

In order to obtain a template for modeling the structure of SlitOBP1, we searched the PDB database using the amino acid sequence of SlitOBP1 as query. There were 35 hits in total and the best one was the A chain of Bombyx mori GOBP2 which showed a con- sistency of $53 \%$, similarity of $72 \%$, E-value of 1.05167E-59, and bit score of 225.713. In addition, the full length of this template protein was 141 amino acids, the resolution was $1.40 \AA$, which meet the requirement of the homologous modeling. Therefore, the solution structure of GOBP in Bombyx mori (PDB code: $2 \mathrm{WCJ}$ ) was selected as template (Fig. 1A) and the 3D model of SlitOBP1 was predicted by homology modeling using the alignment between SlitOBP1 and BmorGOBP. After refinement, the heavy atoms root mean square deviation (RMSD) between the model and the template was $1.5 \AA$ up and down fluctuations, suggesting that the differences between the model and the template in the overall structure were negligible (Fig. 1B). The Verify Score of the final SlitOBP1 model determined by Profiles-3D was 66.29 (Fig. 1C), which is close to the expected verification score of 64.68. The Verify Score of the final SlitOBP1 was above 0, and the Ramachardran plot showed the structure of $99.3 \%$ amino acid residues were in allowed regions (Fig. 1D), implying that the overall quality of the predicted SlitOBP1 structure was reliable.

Six a-helices were present in the predicted 3D structure of SlitOBP1, located between residues 1-24 (A), 33-38 (B), 46-79 (C), 83-101 (D), 107-126 (E) and 134-139 (F). Three pairs of disulfide bridges connected Cys19 and Cys54, Cys50 and Cys108, Cys97 and Cys117 (Fig. 1E). In this model, the hydrophilic residues were mostly present on the surface of the protein, while the hydrophobic residues formed a hydrophobic cavity inside of the protein (Fig. 1F).

\section{Molecular docking}

Based on the principle of five rules in the ISIS BASE, the unreasonable and repetitive compounds were removed and the 1,625,185 compounds were used for the subsequent screening. Five potential binding sites were identified by the Binding site program (Fig. 2). The one was chosen as the binding site for screening for its localization in the hydrophobic cavity, and other binding sites were deviated from the cavity. After evaluated by the five scoring functions, the $20 \%$ default ligands (the ligands could form the complex with the receptor protein) stand for $42 \%$ of the total number. This suggested that the method and parameters setting applied in this screening is rational and efficient. After docking by the Ligandfit, 1,536,550 compounds could be docking successfully with the SlitOBP1. By the established method, the top 20\% compounds ranking by the Consensus Score were selected for the second screening. Subsequently, 23765 out of 23940 compounds were docking successfully by the CDOCKER. The compounds without the CAS numbers were ruled out, and the final 2040 com- 
pounds were selected. Based on the scoring functions, including -CDOCKER interaction energy, -CDOCKER energy, -PLP1 and LigScore1, the ranking top 100 compounds were shown in the Table 1 . These compounds, which could bind to SlitOBP1, displayed a variety of different structures, including terpenoids, linear aldehydes, ketone, and aromatic compounds.
The results showed the SlitOBP1 possesses broad spectra of ligand-binding activities to many compounds in various applications. The result of detailed binding mode of one selected chemical (45234-02-4) with the protein was showed in Fig. 3.
A
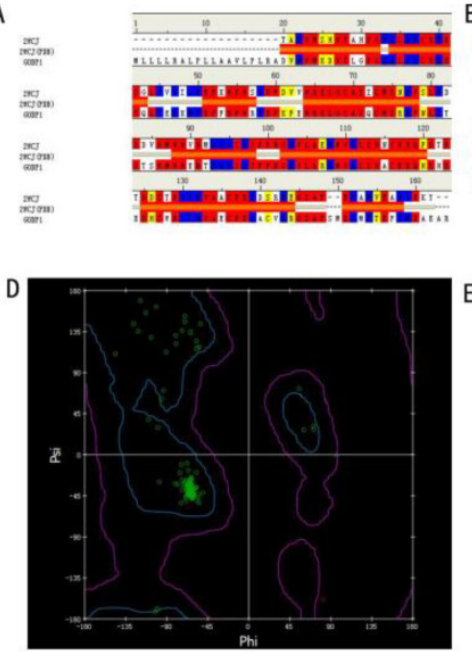
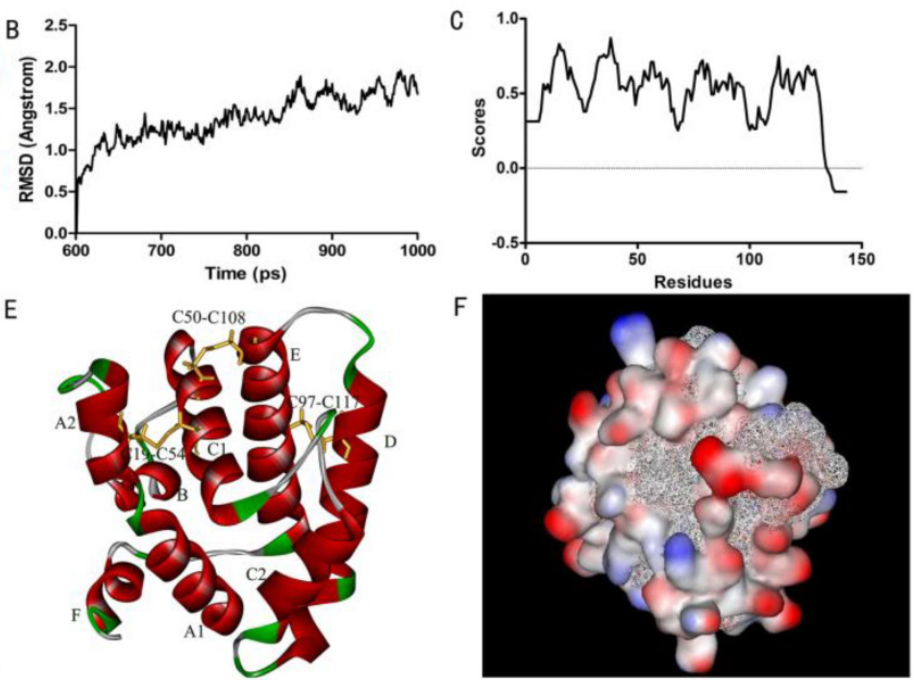

Figure 1. Three-dimensional (3D) model of SlitOBP1. (A)Sequence alignment of SiltOBP1 and BmorOBP. Strictly identical are highlighted with red background. (B) Root-mean square deviation obtained from the $1 \mathrm{~ns}$ molecular dynamics trajectory for SlitOBPI. (C) The Verify Score (Profile-3D) of the protein model of SlitOBPI. Residues with positive compatibility score are reasonably folded. (D) The Ramachandran plot of SlitOBPI. (E) The model of the SlitOBPI. Ribbon represented the selected conformer of the SlitOBP1. The three disulfide bridges are represented as yellow sticks. The secondary structures are labeled. A, B, C, D, E, F showed the six $\alpha$-helices. (F) The hydrophobic and hydrophilic model of the SiltOBPI. The protein was folded and formed a spherical structure. The solid surface of the protein was covered by the hydrophilic residues, while the hydrophobic residues formed a hydrophobic cavity in inside of the protein (the white mesh surface).

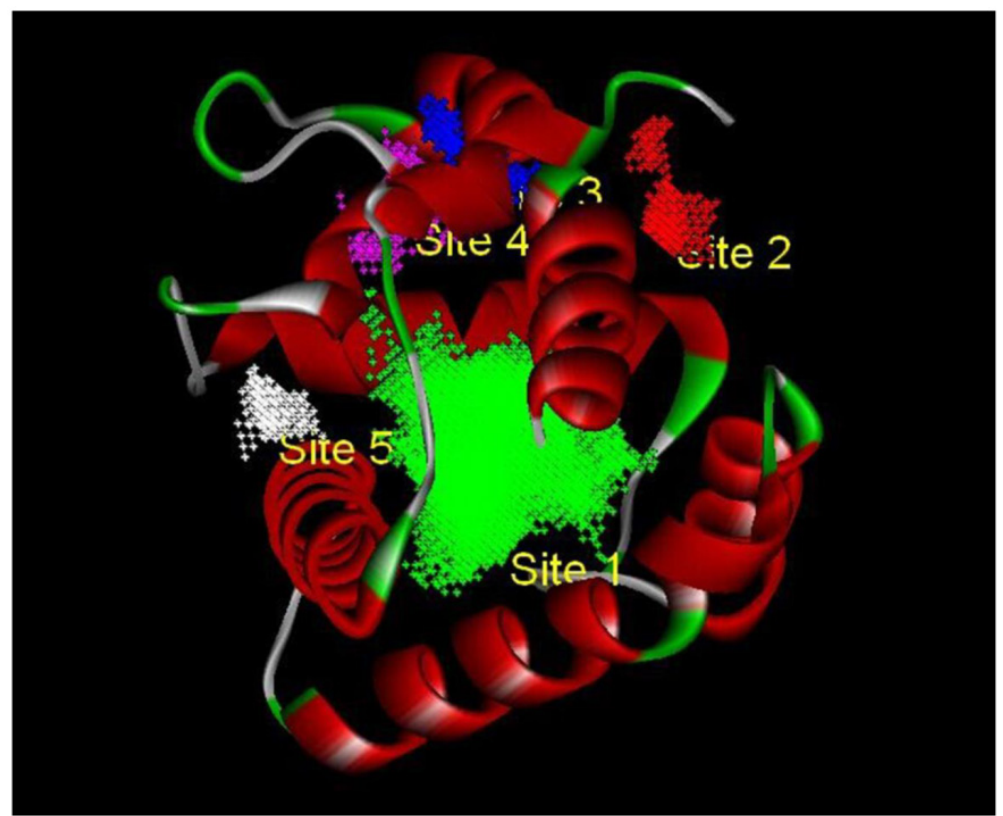

Figure 2. The five binding sites prediction of SlitOBP1. The five binding sites were identified based on the Binding Site Program in DS. The green one was represented as Binding site 1, the red one was for Binding site 2, the blue one was for Binding site 3, the purple one was for Binding site 4 and the white one was for Binding site 5 . 

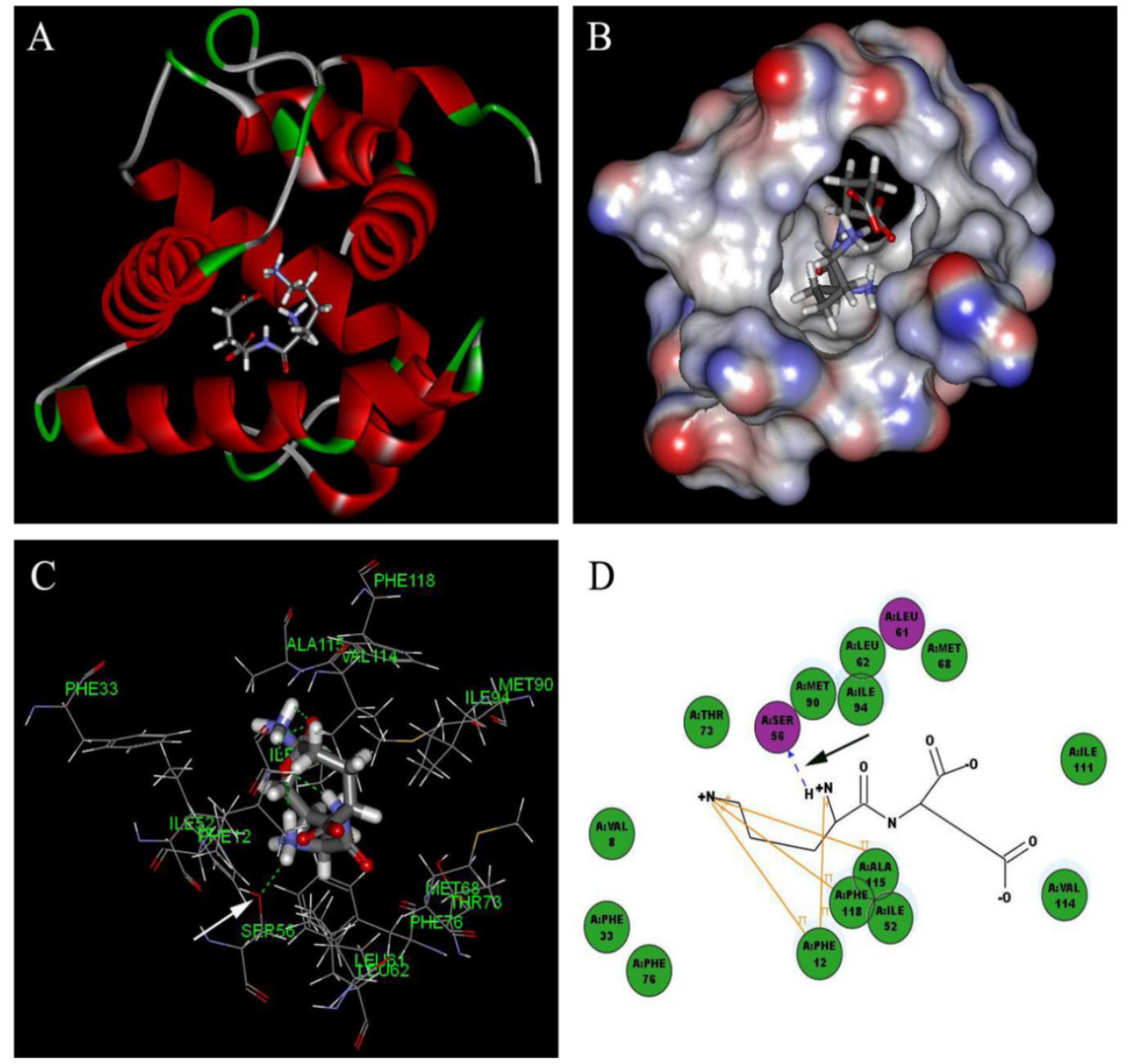

Figure 3. Detailed binding mode of one selected chemical (45234-02-4) with the SlitOBP1. (A) The binding mode between the ligand and SlitOBP1; (B) The position of 45234-02-4 in the binding pocket of SlitOBPI; (C) The 3D binding mode between 45234-02-4 and the key residues in the SlitOBPI; (D) The 2D binding mode between 45234-02-4 and the key residues in the SlitOBPI. The arrows point to the hydrogen bonds, and conjugated effects were showed by yellow lines.

Table 1. Chemicals screened by four score functions in the docking analysis with SlitOBPI.

\begin{tabular}{|c|c|c|c|c|c|c|c|c|c|}
\hline CAS No. & $\begin{array}{l}\text {-CDOCKER } \\
\text { energy }\end{array}$ & $\begin{array}{l}\text {-CDOCKER } \\
\text { Interaction energy }\end{array}$ & -PLP1 & LigScore1 & CAS No. & $\begin{array}{l}\text {-CDOCKER } \\
\text { energy }\end{array}$ & $\begin{array}{l}\text {-CDOCKER } \\
\text { Interaction energy }\end{array}$ & -PLP1 & LigScore1 \\
\hline $375-95-1$ & 5.66456 & 38.6991 & 92.32 & 2.72 & $678-39-7$ & 12.0627 & 40.6552 & 90.46 & 1.29 \\
\hline $754-96-1$ & 14.7061 & 41.5673 & 93.99 & 2.88 & $865-79-2$ & 8.13773 & 39.7778 & 91.82 & 2.83 \\
\hline $2342-09-8$ & 10.4978 & 35.2783 & 87.04 & 2.52 & $2545-89-3$ & 65.1184 & 43.2304 & 76.22 & 4.07 \\
\hline $5545-52-8$ & 53.468 & 43.9979 & 80.33 & 1.31 & $5891-53-2$ & 70.7165 & 42.5603 & 72.4 & 2.45 \\
\hline 6099-08-7 & 58.9235 & 40.5716 & 72.36 & 2.18 & $10567-86-9$ & 23.4187 & 32.8927 & 70.36 & 3.45 \\
\hline $15080-84-9$ & 49.5768 & 41.7062 & 70.14 & 2.14 & $17355-09-8$ & 53.8239 & 43.7485 & 70.44 & 2.85 \\
\hline 20556-13-2 & 58.3522 & 41.7623 & 69.74 & 3.28 & $23680-31-1$ & 48.4742 & 41.8875 & 69.35 & 1.45 \\
\hline $24305-27-9$ & 42.031 & 48.771 & 79.74 & 2.5 & $30670-30-5$ & 14.3854 & 40.0041 & 89.06 & 2.53 \\
\hline $42406-77-9$ & 42.2214 & 37.7094 & 76.02 & 1.5 & $45234-02-4$ & 70.5762 & 38.7205 & 72.96 & 2.97 \\
\hline $157843-41-9$ & 9.39257 & 42.3274 & 82.27 & 3.41 & 203303-01-9 & 15.3247 & 37.8305 & 85.54 & 2.59 \\
\hline $430447-82-8$ & 49.3832 & 39.6555 & 76.22 & 1.91 & $145-13-1$ & -17.066 & 48.341 & 75.36 & 3.31 \\
\hline $298-25-9$ & -6.811 & 51.013 & 67.29 & 3.45 & $974-23-2$ & -13.646 & 48.085 & 68.93 & 3.29 \\
\hline $97970-92-8$ & 44.108 & 46.643 & 75.11 & 3.6 & 18797-79-0 & -1.93 & 48.604 & 83.49 & 2.3 \\
\hline $50-24-8$ & -29.79 & 47.565 & 77.92 & 2.96 & $53-03-2$ & -17.895 & 48.297 & 77.04 & 3.26 \\
\hline $3546-11-0$ & 25.128 & 43.484 & 77.76 & 3.83 & $898792-70-6$ & 21.233 & 42.774 & 82.88 & 1.89 \\
\hline $5220-98-4$ & 25.443 & 49.026 & 75.47 & 2.46 & $474-45-3$ & -7.417 & 47.362 & 75.51 & 3.15 \\
\hline $3788-44-1$ & 57.871 & 46.901 & 75.49 & 3.79 & 516-54-1 & -5.032 & 49.098 & 76.06 & 3.11 \\
\hline $37481-42-8$ & 31.588 & 45.445 & 82.44 & 1.98 & $75522-73-5$ & 11.787 & 46.752 & 78.41 & 2.06 \\
\hline $655256-70-5$ & 24.284 & 45.997 & 76.16 & 3.6 & $80-75-1$ & -13.706 & 46.877 & 77.04 & 3.18 \\
\hline $1923-27-9$ & 1.291 & 48.813 & 78.64 & 2.77 & $2681-55-2$ & -9.172 & 46.323 & 76.15 & 3.13 \\
\hline 596-69-0 & -70.85 & 44.134 & 75 & 2.73 & $975-53-1$ & -26.872 & 49.709 & 79.06 & 3.36 \\
\hline $103335-41-7$ & -5.825 & 48.346 & 78.29 & 2.84 & $4777-62-2$ & -20.033 & 50.85 & 80.3 & 3.29 \\
\hline $1239-31-2$ & 5.09 & 47.713 & 80.84 & 2.71 & 23930-19-0 & -1.695 & 50.498 & 75.75 & 3.31 \\
\hline $1482-78-6$ & -4.581 & 48.931 & 79.12 & 2.84 & $1600-76-6$ & -9.031 & 46.425 & 81.35 & 2.72 \\
\hline
\end{tabular}




\begin{tabular}{|c|c|c|c|c|c|c|c|c|c|}
\hline $38398-44-6$ & -7.135 & 51.478 & 80.82 & 3.11 & $10388-62-2$ & -71.942 & 44.895 & 75.7 & 2.63 \\
\hline $3684-84-2$ & -3.216 & 45.385 & 82.21 & 3.25 & $22733-60-4$ & -10.97 & 46.621 & 76.02 & 3.11 \\
\hline $35100-44-8$ & -18.866 & 48.178 & 79.19 & 2.95 & $19427-36-2$ & -7.934 & 47.701 & 80.58 & 3.24 \\
\hline 103303-35-1 & -23.264 & 49.377 & 79.87 & 3.2 & $82419-52-1$ & 31.319 & 42.116 & 76.37 & 3.13 \\
\hline $264218-23-7$ & 5.133 & 40.165 & 78.34 & 2.83 & $566-42-7$ & -17.567 & 48.278 & 80.88 & 3.82 \\
\hline 641-78-1 & -13.068 & 47.328 & 76.24 & 2.79 & $1000776-27-1$ & 46.836 & 52.262 & 80.15 & 2.65 \\
\hline $143691-84-3$ & 14.767 & 49.475 & 79.53 & 4.08 & 75263-33-1 & 39.288 & 49.301 & 77.73 & 2.43 \\
\hline $56258-32-3$ & 21.974 & 44.602 & 84.84 & 3.43 & $1241-87-8$ & 18.77 & 45.665 & 80.06 & 3.82 \\
\hline $124858-35-1$ & 30.042 & 48.353 & 89.21 & 2.7 & 71795-44-3 & 35.502 & 47.371 & 80.02 & 2.33 \\
\hline $317807-10-6$ & 19.695 & 42.542 & 78.37 & 2.43 & $75530-60-8$ & 13.451 & 51.474 & 83.8 & 3 \\
\hline 806-29-1 & -18.711 & 45.22 & 81.24 & 3.01 & $433690-46-1$ & 35.43 & 49.76 & 81.93 & 3.11 \\
\hline $533922-44-0$ & 34.501 & 46.763 & 82.18 & 3.64 & $571-67-5$ & -3.522 & 43.374 & 82.71 & 2.58 \\
\hline $532940-59-3$ & 26.991 & 47.063 & 85.01 & 2.93 & $435282-71-6$ & 31.114 & 45.625 & 80.64 & 3.52 \\
\hline 34061-33-1 & 10.708 & 38.643 & 70.23 & 2.14 & 913835-39-9 & 32.066 & 39.347 & 72.31 & 3.31 \\
\hline $957060-97-8$ & 31.913 & 38.511 & 70.17 & 3.27 & 72666-14-9 & 30.778 & 39.451 & 69.72 & 3.93 \\
\hline $520-17-2$ & 23.067 & 37.483 & 68.93 & 2.71 & $72617-60-8$ & -15.525 & 38.467 & 68.02 & 2.83 \\
\hline 1881-37-4 & 0.168 & 38.988 & 71.87 & 2.81 & 331759-58-1 & 36.648 & 38.6 & 75.12 & 3.03 \\
\hline 67034-83-7 & -15.668 & 43.242 & 71.73 & 3.1 & $946049-56-5$ & 31.732 & 38.083 & 76.39 & 2.96 \\
\hline $3569-77-5$ & 28.344 & 38.857 & 68.79 & 3.35 & $4747-99-3$ & 38.789 & 42.468 & 67.33 & 3.47 \\
\hline $21178-57-4$ & -0.063 & 39.578 & 74.35 & 3.57 & $145915-60-2$ & 2.558 & 37.71 & 83.67 & 3.36 \\
\hline $5189-96-8$ & -5.336 & 40.639 & 69.93 & 3.21 & $89875-86-5$ & -20.632 & 36.333 & 69.09 & 2.66 \\
\hline 38734-38-2 & 12.374 & 43.012 & 76.64 & 2.28 & $98067-16-4$ & 27.058 & 44.607 & 66.55 & 2.67 \\
\hline $53-43-0$ & -15.654 & 43.182 & 71.73 & 3.1 & $175448-32-5$ & -9.385 & 36.938 & 69.51 & 3.13 \\
\hline $532940-59-3$ & 27.684 & 45.391 & 85.01 & 2.93 & 913835-39-9 & 32.066 & 39.347 & 72.31 & 3.31 \\
\hline $21178-57-4$ & -0.66 & 38.778 & 67.46 & 3.33 & 530133-81-4 & 20.029 & 39.874 & 91.04 & 2.98 \\
\hline $161987-77-5$ & 10.727 & 36.739 & 78.46 & 2.32 & $36271-80-4$ & -4.822 & 41.479 & 77.9 & 3.07 \\
\hline
\end{tabular}

\section{Fluorescence Binding Assays}

The SlitOBP1 was induced and expressed successfully $(21.9 \mu \mathrm{g} / \mu \mathrm{l})$. The purified protein was resolved as a single band with molecular weight of 25 $\mathrm{kDa}$ as determined by SDS-PAGE and Western blot (Fig. 4A (a)\&(b)). By titrating SlitOBP1 with increasing concentration of 1-NPN, a saturation and linear Scatchard plot (Fig. 4B) were observed. The SlitOBP1 could bind to the probe with a dissociation constant of $3.237 \pm 0.20 \mu \mathrm{M}$ (Fig. 4B). By using 1-NPN as the fluorescent reporter, the affinity of SlitOBP1 to a series of compounds was measured (Fig. 4C\&D). The $\mathrm{IC}_{50}$ values (the concentration of the ligand that yielded $50 \%$ of the initial fluorescence value) and calculated binding constants were reported in Table 2. The SlitOBP1 showed high affinities with most of the tested compounds, including 375-95-1, 50-24-8, 53-03-2 and 865-79-2, while some chemicals showed no binding potential to the receptor protein, such as 2342-09-8 and 30670-30-5.

Table 2. Key residues of the interaction between SlitGOBPI and candidate compounds.

\begin{tabular}{ll}
\hline Interaction & Key residues \\
\hline $\begin{array}{l}\text { VDW interacton } \\
\text { energy }\end{array}$ & $\begin{array}{l}\text { Thr9, Phe12, Ile52, Met68, Ile94, Phe118, Leu61, Leu62, } \\
\text { Phe12, Phe36, Phe33, IAla115, Trp37, Val114 } \\
\begin{array}{l}\text { Electrostatic in- } \\
\text { teracton energy }\end{array} \\
\begin{array}{l}\text { Arg110, Ser56, Thr73, Thr9, Arg110, Met5, Glu98 } \\
\text { conjugated effect }\end{array}\end{array}$ \\
\hline
\end{tabular}

The marked bold represented the selected key residues for the following experiment.

\section{Key residues and simulation of site-directed mutagenesis}

The structural studies provided insight into the ligand-binding mechanism of SlitOBP1. During the binding of SlitOBP1 with various ligands, the hydrogen bond, van der Waals interactions, electrostatic force and conjugative effect were crucial for binding activities. After docking with the screened compounds, nine amino acids of SlitOBP1 were identified as key residues for the ligand binding specificity (Table 3). The nine key amino acids were mutated to alanine, respectively, and the binding activities of each mutant to the 21 compounds were evaluated. The result showed the Phe12, Ile52, Ile94 and Phe118 could affect the formation of Van der Waals interactions, while Thr9, Trp37, Ser56, Glu98 and Arg110 could affect the formation of electrostatic force and hydrogen bond, eventually could affect the conformation of the cavity to the final binding affinities of the protein. The results showed majority of the scores of binding activities of mutations were reduced significantly, which suggested these key residues could be responsible in regulating the binding activities of the SlitOBP1 to the compounds. The Table 4 showed the docking scores of partial compounds by comparison of the wild type model and mutant model. The analysis of $1 \mathrm{~ns}$ molecular dynamics revealed that these complexes of SlitOBP1 and compounds were stable, which suggested the obtained result was reliable (Fig.5A\&B). 
Table 3. Fluorescence competitive binding affinities of selected components to recombinant SlitOBP1.

\begin{tabular}{|c|c|c|c|}
\hline \multirow[t]{2}{*}{ Ligands } & \multirow[t]{2}{*}{ CAS } & \multicolumn{2}{|c|}{ SlitOBP1 } \\
\hline & & $\mathrm{IC}_{50}$ & $\mathrm{~K}_{\mathrm{i}}$ \\
\hline \multicolumn{4}{|l|}{ Alcohols } \\
\hline 1H,1H,2H,2H-perfluoro-1-decanol & $678-39-7$ & 5.97 & 3.69 \\
\hline $1 \mathrm{H}, 1 \mathrm{H}, 10 \mathrm{H}, 10 \mathrm{H}$-perfluoro-1,10-decanediol & $754-96-1$ & 6.68 & 4.13 \\
\hline $1 \mathrm{H}, 1 \mathrm{H}, 9 \mathrm{H}, 9 \mathrm{H}$-perfluoro-1,9-nonanediol & 203303-01-9 & 9.45 & 5.84 \\
\hline \multicolumn{4}{|l|}{ Acids } \\
\hline Perfluorononanoic acid & $375-95-1$ & 6.08 & 3.76 \\
\hline 9-Chlorohexadecafluorononanoic acid & $865-79-2$ & 6.00 & 3.73 \\
\hline H-Lys-Glu-OH & $45234-02-4$ & 5.86 & 3.62 \\
\hline H-Glu-Lys-OH & $5891-53-2$ & - & - \\
\hline H-Gly-Tyr-Gly-OH & $6099-08-7$ & 6.11 & 3.78 \\
\hline H-Phe-Met-OH & $15080-84-9$ & - & - \\
\hline H-Lys-Ile-OH & $20556-13-2$ & 7.52 & 4.65 \\
\hline H-Tyr-Glu-OH & $2545-89-3$ & - & - \\
\hline N-Cbz-L-Aspartic acid 4-tert-butyl ester & $5545-52-8$ & 6.70 & 4.14 \\
\hline \multicolumn{4}{|l|}{ Ketones } \\
\hline 1,2-Dehydrocortisone & $53-03-2$ & 5.00 & 3.09 \\
\hline Delta-5-pregnenolone & $145-13-1$ & 14.77 & 9.13 \\
\hline $\begin{array}{l}11 \beta, 17 a, 21 \text {-trihydroxypregna-1,4-diene-3,20- } \\
\text { dione }\end{array}$ & $50-24-8$ & 5.02 & 3.10 \\
\hline \multicolumn{4}{|l|}{ Heterocyclic compounds } \\
\hline 16,17-epoxypregnenolone & $974-23-2$ & 8.28 & 5.12 \\
\hline Corynoline & $18797-79-0$ & - & - \\
\hline Pyr-His-Pro-NH2 & $24305-27-9$ & 7.18 & 4.44 \\
\hline L-Tyrosine benzyl ester & $42406-77-9$ & 7.23 & 4.47 \\
\hline \multicolumn{4}{|l|}{ Others } \\
\hline Perfluorosebaconitrile & $2342-09-8$ & - & - \\
\hline $1 \mathrm{H}, 1 \mathrm{H}, 2 \mathrm{H}, 2 \mathrm{H}$-perfluorodecylamine & $30670-30-5$ & - & - \\
\hline
\end{tabular}

\section{Site-directed mutagenesis of SlitOBPI and binding specificities of mutants}

By the site-directed mutagenesis, the residues (Phe12, Ile52, Thr9, Trp37, Glu98) were replaced with the alanine. When analyzed by SDS-PAGE, the expressed mutant protein showed band with similar molecular weight with wild-type protein (Supplementary Fig. 1). Probed by 1-NPN, the saturation and linear Scatchard plot was observed, and saturation binding curves analysis revealed that the dissociation constants for 1-NPN to mutants Phe12, Ile52, Thr9, Glu98 and Trp37 were $6.78 \pm 0.25 \mu \mathrm{M}, 5.43 \pm 0.31 \mu \mathrm{M}$, $4.21 \pm 0.19 \mu \mathrm{M}, 3.67 \pm 0.25 \mu \mathrm{M}$ and $4.54 \pm 0.28 \mu \mathrm{M}$, respectively. Affinities of the mutant proteins to these 21 chemicals were measured by competitive binding assays. The result showed that, after mutanting the key amino acids, many ligands could not compete with $50 \%$ of the 1-NPN even when their concentration reached $20 \mu \mathrm{M}$. Interestingly, in mutant Phe12 and Ile52, all the ligands could not bind to the OBP1. As predicted by computer simulation, most of the ligands showed poor binding affinities to the mutants, while some chemicals also showed better binding activities, such as the 6099-08-7 with mutant Thr9, mutant Glu98 and 678-39-7 with mutant Trp37 (Fig. 6).

Table 4. comparison between the docking score using original model and mutant model.

\begin{tabular}{|c|c|c|c|c|c|c|c|c|}
\hline $\begin{array}{l}\text { Mutant } \\
\text { amino acid }\end{array}$ & CAS No. & $\begin{array}{c}\text { Decreasing auplitude of } \\
\text { score after mutant (\%) }\end{array}$ & $\begin{array}{l}\text { Mutant } \\
\text { amino acid }\end{array}$ & CAS No. & $\begin{array}{c}\text { Decreasing auplitude of } \\
\text { score after mutant (\%) }\end{array}$ & $\begin{array}{l}\text { Mutant } \\
\text { amino acid }\end{array}$ & CAS No. & $\begin{array}{c}\text { Decreasing auplitude of } \\
\text { score after mutant (\%) }\end{array}$ \\
\hline \multirow[t]{13}{*}{ Phe12 } & $42406-77-9$ & 7.37 & & $974-23-2$ & 15.38 & & 53-03-2 & 19.11 \\
\hline & $974-23-2$ & 64.76 & & $754-96-1$ & 39.70 & & $50-24-8$ & 12.82 \\
\hline & $754-96-1$ & 40.04 & & $53-03-2$ & -0.73 & & $2545-89-3$ & 3.67 \\
\hline & 53-03-2 & 27.54 & & $50-24-8$ & 6.08 & & $6099-08-7$ & -6.84 \\
\hline & $50-24-8$ & 13.70 & & $2545-89-3$ & 3.06 & & $865-79-2$ & 45.33 \\
\hline & $2545-89-3$ & 4.15 & & $6099-08-7$ & -9.06 & Trp37 & 754-96-1 & 23.66 \\
\hline & 20556-13-2 & 4.15 & & 20556-13-2 & 0.02 & & $53-03-2$ & 37.71 \\
\hline & $45234-02-4$ & 5.84 & & $45234-02-4$ & -0.61 & & $50-24-8$ & 17.79 \\
\hline & $24305-27-9$ & 14.87 & & $24305-27-9$ & 13.82 & & $2545-89-3$ & 5.59 \\
\hline & $678-39-7$ & 48.92 & & $678-39-7$ & 29.77 & & $6099-08-7$ & -0.63 \\
\hline & $375-95-1$ & 112.54 & & $375-95-1$ & 16.61 & & $678-39-7$ & -0.41 \\
\hline & $5545-52-8$ & 13.67 & & $5545-52-8$ & 1.55 & & $5545-52-8$ & 6.64 \\
\hline & $865-79-2$ & 43.73 & & $865-79-2$ & 20.39 & & $865-79-2$ & 38.21 \\
\hline \multirow[t]{12}{*}{ Ile52 } & $974-23-2$ & 17.22 & Phe118 & $42406-77-9$ & -0.69 & Ser56 & 42406-77-9 & -2.34 \\
\hline & $754-96-1$ & 27.26 & & $974-23-2$ & 20.37 & & $2545-89-3$ & -0.11 \\
\hline & 53-03-2 & 26.82 & & $754-96-1$ & 26.04 & & $6099-08-7$ & -2.53 \\
\hline & $50-24-8$ & 7.82 & & $53-03-2$ & 45.92 & & 20556-13-2 & 6.19 \\
\hline & $2545-89-3$ & -0.58 & & $50-24-8$ & 15.24 & & $45234-02-4$ & 2.20 \\
\hline & $6099-08-7$ & 0.68 & & $6099-08-7$ & -1.05 & & $24305-27-9$ & 5.38 \\
\hline & $45234-02-4$ & 3.07 & & 20556-13-2 & 6.51 & Glu98 & $974-23-2$ & 36.12 \\
\hline & $24305-27-9$ & 2.02 & & $24305-27-9$ & 1.86 & & $2545-89-3$ & 2.63 \\
\hline & $678-39-7$ & 17.08 & & $678-39-7$ & 6.38 & & $6099-08-7$ & -9.45 \\
\hline & $375-95-1$ & 44.88 & & $375-95-1$ & 30.92 & & $375-95-1$ & -82.33 \\
\hline & $5545-52-8$ & 5.24 & & $5545-52-8$ & 2.77 & Arg110 & $754-96-1$ & -10.06 \\
\hline & $865-79-2$ & 79.73 & & $865-79-2$ & 35.38 & & 53-03-2 & 35.25 \\
\hline Ile94 & $42406-77-9$ & -11.82 & Thr9 & $754-96-1$ & 1.63 & & $50-24-8$ & 6.95 \\
\hline
\end{tabular}



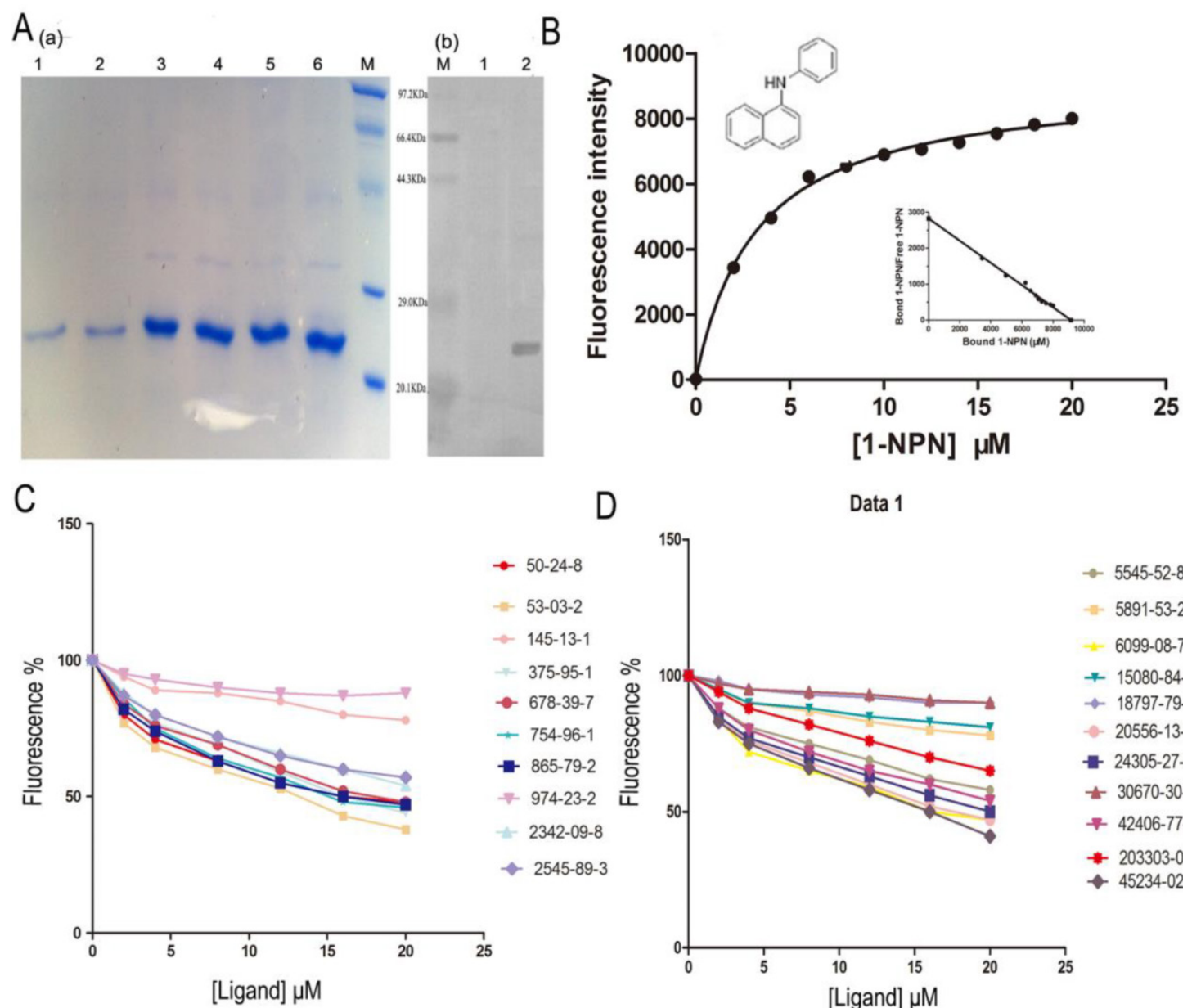

D

Data 1
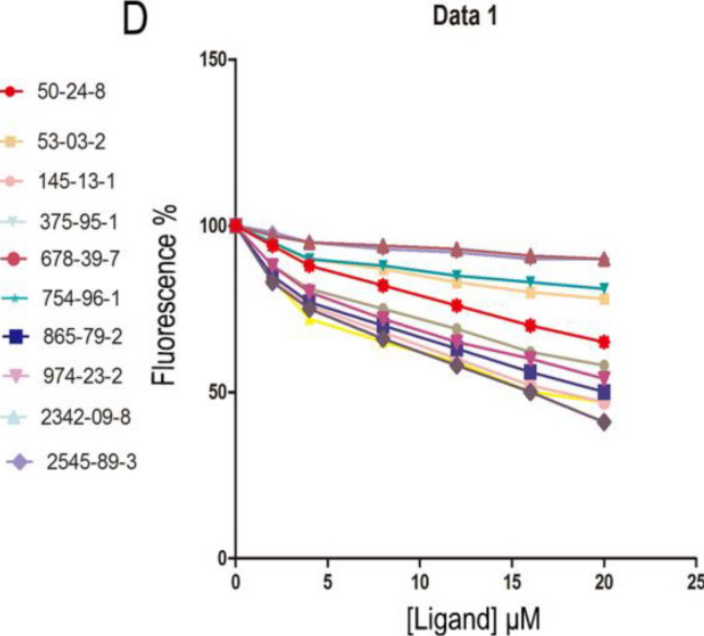

Figure 4. Competitive binding study of SlitOBPI to a series of selected compounds. (A) Expression and purification of SlitOBPI. (a) The blot showed one specific band at around $25 \mathrm{KDa}$ in agreement with the predicted value. SlitOBPI was induced and expressed in pET-28a vector. Purification was accomplished by HisTrap affinity columns; Lane 1-2 showed the protein after renaturation; Lane 3-6 showed purified inclusion body of the protein. M: molecular weight marker of 20.1, 29.0, 44.3, 66.4 and 97.3 KDa. (b) Western blot analysis of the SlitOBP. Immunoblotted with serum (diluted 1: 2000) and visualized by ECL. M: molecular weight marker of 20.1, 29.0, 44.3, 66.4 and 97.3 KDa. 1: control. 2: Purified protein. (B) The binding curve of 1-NPN and relative Scatchard plot analysis (inset). To measure the affinity of 1-NPN to SlitOBPI, the fluorescence of $2 \mu M$ 1-NPN in $50 \mathrm{mM}$ Tris- $\mathrm{HCl}$ was excited at $337 \mathrm{~nm}$ and emission spectra were recorded between $350 \mathrm{~nm}$ and $480 \mathrm{~nm}$. Then, $2 \mu M$ of protein was added and titrated with aliquots of $1 \mathrm{mM}$ 1-NPN to final concentrations of 2 to $20 \mu \mathrm{M}$. The experiment was replicated for at least three times, and the data were analyzed using Prism software and indicated the presence of a single binding site. The dissociation constant was $3.237( \pm 0.20$ SEM). (C)\&(D) The solution was excited at $337 \mathrm{~nm}$. Competitive binding of the selected ligands with SlitOBPI. Final solutions of $2 \mu \mathrm{M}$ SlitOBPI protein and $2 \mu \mathrm{M} 1$-NPN were titrated with $1 \mathrm{mM}$ solution of each ligand in methanol to final concentrations of 0-20 $\mu \mathrm{M}$. The figure reported averages of three replicates.

\section{Discussion}

Far and wide, the inspired biosensor offers potential applications for environmental monitoring, agriculture, anti-bioterrorism, disease diagnostics, and food safety [36]. The intrinsic properties of OBPs make them excellent candidates for biosensors [17]. Ramoni et al [37] investigated the possibility of utilizing a mutant of OBP as nano-biosensors for the indication of hazardous compounds. Pietrantonio et al [13] presented biosensors for vapor phase detection based on surface acoustic wave resonators coated with OBPs [17], and a biosensor based on OBP was designed with its ligands detected by electrochemical impedance sensing [12]. The application of these proteins in ligands detection is very appealing, and the focus of this study is to explore an insect OBP on the basis of its broad binding activities. Moreover, the design of mutants with desired characteristics enables more specific and efficient detections.

The way in which organisms detect volatile compounds within the environment has been of interest to scientists for decades [38]. For their crucial role of OBPs in olfaction, structural properties are the main target of future investigations. Homology modeling is an efficient method for 3D construction, which can provide a structural basis for evaluating and designing ligand sensors [36]. As demonstrated in this study, the SlitOBP1 is composed of six a-helical domains arranging in a very compact structure, which encloses a hydrophobic cavity. Compared to vertebrate OBPs, the presence of three interlocked disulphide bridges in insect OBPs confers limited flexibility to the structure, which prevents insect OBP from thermal denaturation and proteolysis [19]. This long C terminus structure of SlitOBP1 may be enough to 

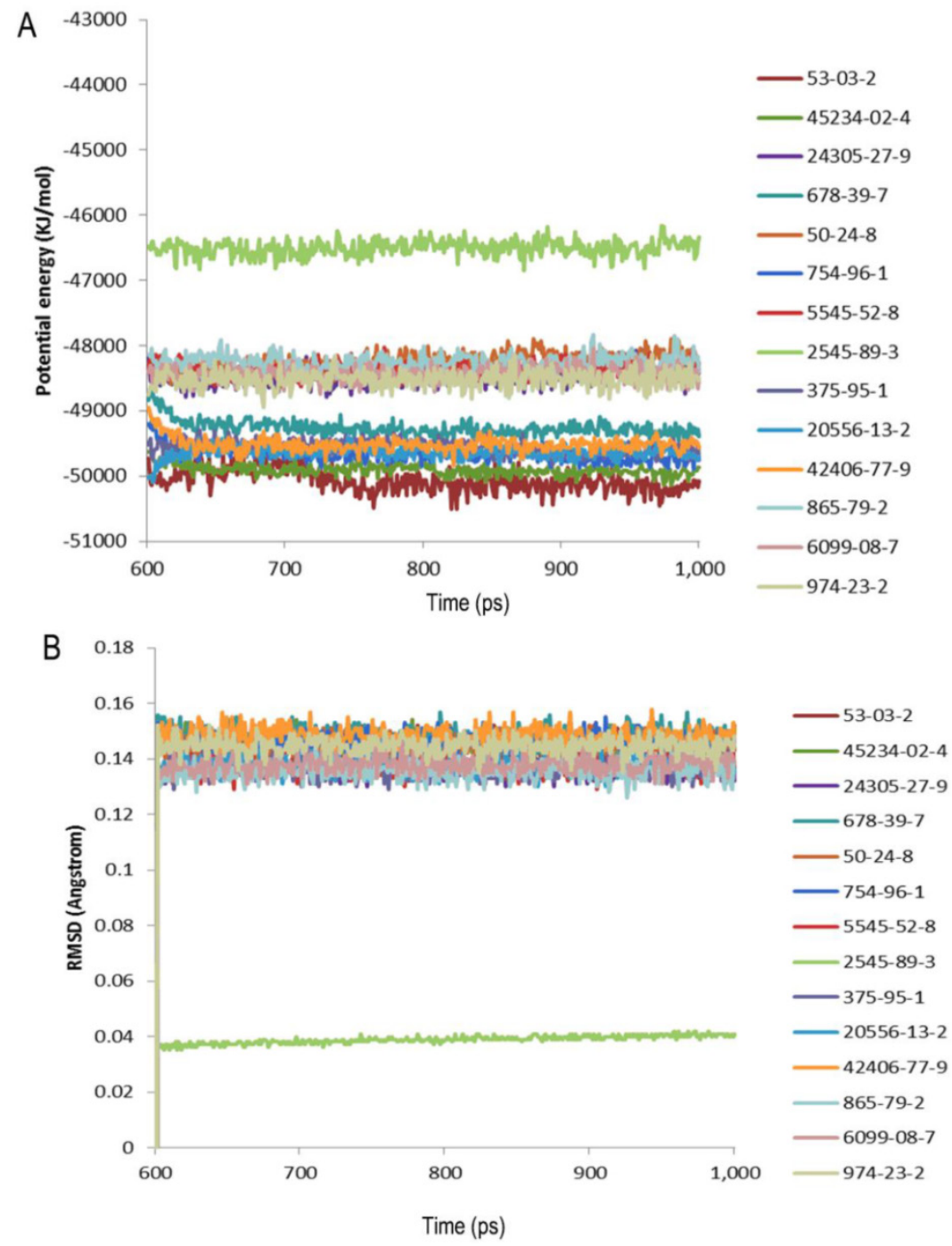

Figure 5. Evaluation of the complexes by molecular dynamics. (A) Potential energy with respect to simulation time for Ins molecular dynamic of the complex of SlitGOBPI with partial selected candidates; (B) Root-mean square deviation obtained from the Ins molecular dynamics trajectory for the complex of SlitGOBPI with partial selected candidates.

form the seventh a-helix as previously mentioned $[20,38]$, which could form a "molecular switch" at low $\mathrm{pH}$ to provide a fast, efficient way of releasing the ligand and restoring the functionality of the biosensor element [19].

The OBPs could be presented as ideal sensor material due to their versatile sensing and detecting abilities. The identification of potential binding modes and poses can be obtained by the molecular docking accurately [40]. To investigate the ligand binding process, the molecular recognition was simulated by molecular docking [41]. Based on the conformation of protein and ligands, the detailed binding modes were doped out. The method described previously in the Cheng [42] was applied for the parameter settings in the Ligandfit, and the results showed the binding efficiency was depended on the number of Monte Carlo Trials and different energy grid. For screening the chemicals in the first round by the Ligandfit, the scoring function, the PLP1 showed better evaluation ability than others. By taking the PLP1 scoring function as the prior evaluation, the number of chemicals is reduced from $1,536,550$ to 23,940 by the first round screening, only accounting for 1/64 of the original number. Thus, the molecular docking could be applied for eliminating improper ligands [36]. Instead of reducing the number of the chemical, the aim of the second round screening is to identify the chemicals which have better and more rational binding mode with the protein. Although the simulation method applied here could not identify the best binding candidate, it still represents a reliable, convenient and rapid way to screen the candidate chemicals. 23765 out of 1,625,185 compounds were found to have good affinities with SlitOBP1, including aliphatic alcohols, ketones, aldehydes, esters and acid, as well as heterocyclic and aromatic compound derivatives. It is significant to investigate the dynamic state by the molecular dynamics simulation considering the ligand and the receptor protein as a complex as all the proteins are dynamic in solution and have structural flexibility. In this study, the complexes of the protein and the compounds were investigated by the 1ns molecular dynamics simulation. The potential energy and root-mean square deviation showed the compounds could form stable complex with the protein. The fact that SlitOBP1 can bind to large number of natural and synthetic structurally unrelated organic compounds suggests that the protein can be applied to detect diverse chemicals in many fronts, including threatening compounds for human health in the air and in water, volatile organic compounds (VOCs) or chemicals have potential impact on climate and long-term health effect.

To validate the reliability of simulation docking, the top 21 ligands of different structure and rational binding modes appeared in four scoring functions were selected to be the candidate ligands in the competitive binding assay. In the previous studies, the compounds used as binding candidate ligands with the tested OBPs were sex pheromones and plant volatiles, and the aim is at illuminating the role of OBPs 
in the perception of such chemicals in insect olfactory system $[43,44]$. To our knowledge, many compounds investigated here were the first time used to evaluate the use of OBP as a biosensor. These compounds were selected based on their relevance to environment, biology and biosensor, etc. For example, the chemical 678-39-7 was reported to have the ability to combine bioaccumulative potential, toxic effects and extreme persistence [45], the chemical 375-95-1 was reported to be water surface-active fluorocarbon derivatives [46], the 45234-02-4 was an excitatory transmitter of major significance in the mammalian central nervous system [47], the chemical 53-03-2 was applied for prostate cancer [48].

Consistent with the result of the simulation docking, the SlitOBP1 could reversibly bind to most of identified chemicals in the competitive fluorescence binding assays. A number of compounds, namely, 53-03-2, 45234-02-4, 375-95-1, 865-79-2 and 50-24-8, could effectively displace 1-NPN, suggesting that these compounds have high affinities with SlitOBP1. In line with results in the simulation docking, most of the selected compounds could bind to the protein. The
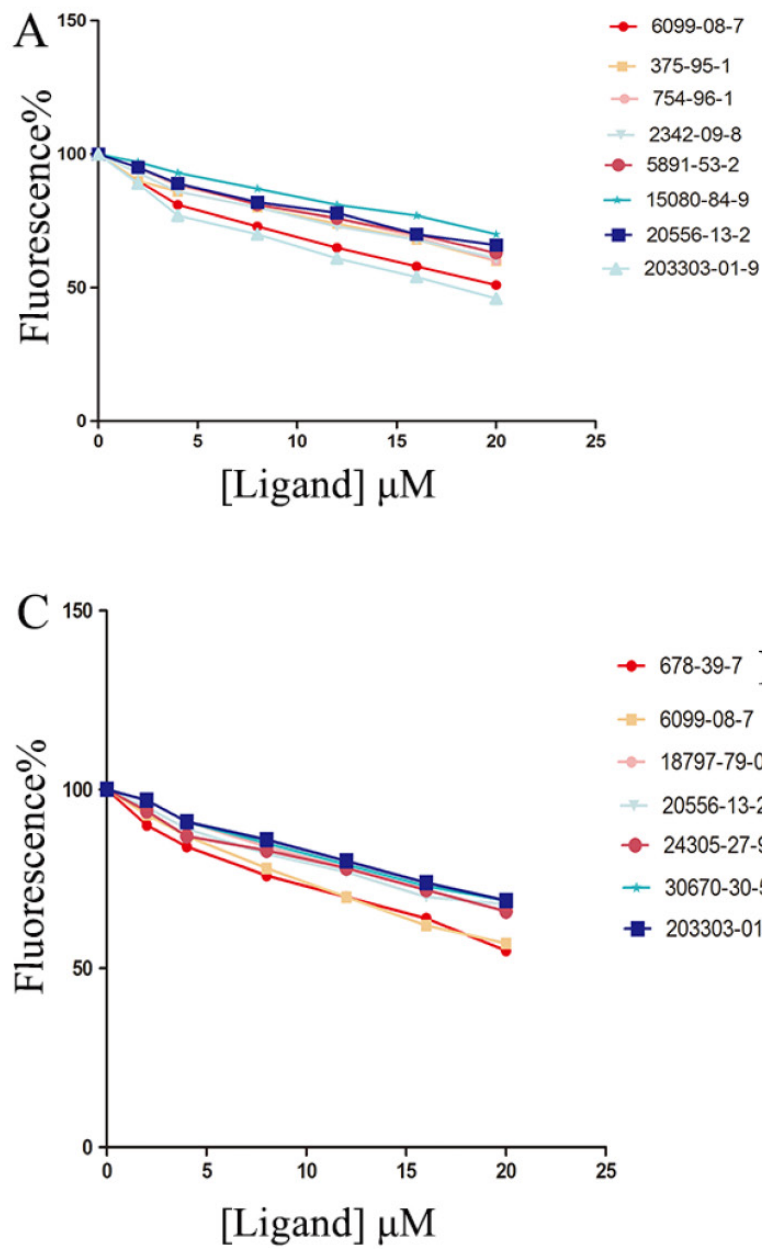

only three exceptions are 2343-09-8, 30670-3-5 and 15080-84-9, which may be due to the strengthening the fluorescence emission by Trp109 on the surface of the protein. Generally speaking, the aliphatic compounds and ketone showed better consistency, while the heterocyclic compounds and chemicals with halogen showed opposite results against previous demonstrated in the simulation docking. This may be due to the complex structure and the formation of other covalent coordination bond. The consistency of the most tested chemical compounds between the simulation docking and experimental data suggested the result of the molecular docking was reliable and the method could be applied in the future screening to save manpower and time. Moreover, the results demonstrated the OBP possesses board affinities to bind and detect various chemicals. The broad binding activities of OBP is presumably due to the peculiar characteristics of its ligand binding site, i.e., a hydrophobic cavity lined with hydrophobic amino acid residues. The fact that the 20 lipophilic amino acids exist inside the protein may explain its binding activities to some lipid solubility compounds.

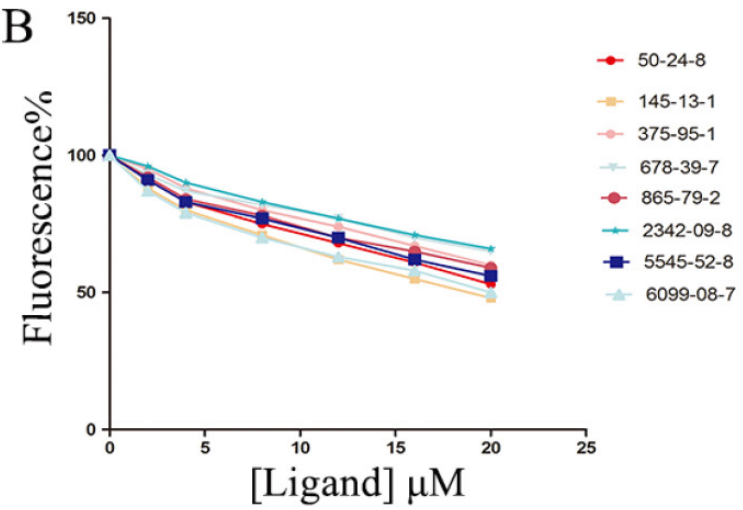

Binding affinities of SlitOBP1 mutants

\begin{tabular}{|c|c|c|c|c|c|c|}
\hline \multirow{2}{*}{$\rightarrow$ 678-39-7 D } & \multirow[t]{2}{*}{ Lignads } & \multicolumn{5}{|c|}{ Ki (AN) } \\
\hline & & Phe12 & Dles2 & Thr19 & Glogs & $\operatorname{Irp37}$ \\
\hline \multirow{3}{*}{$-6099-08-7$} & $50-248$ & $\cdot$ & . & . & 3.58 & . \\
\hline & $53-03.2$ & . & . & . & . & . \\
\hline & $145 \cdot 13.1$ & . & . & . & 2.4 & . \\
\hline \multirow{2}{*}{ - 18797-79-0 } & $375.956-1$ & . & . & 6.45 & 6.19 & . \\
\hline & 678.39 .9 & . & . & . & 6.40 & 3.43 \\
\hline $20556-13-2$ & $754 \cdot 96-1$ & . & . & 8.84 & . & . \\
\hline \multirow{2}{*}{ - 24305-27-9 } & 865.79 .2 & - & . & . & 6.31 & . \\
\hline & 974.23 .2 & . & . & . & . & . \\
\hline \multirow{2}{*}{$\approx 30670-30-5$} & $2442-09.8$ & . & . & 15.51 & 5.95 & . \\
\hline & $2545-99.3$ & . & . & . & - & . \\
\hline \multirow{10}{*}{$-203303-01-9$} & 5845.52 .8 & - & - & - & 5.68 & - \\
\hline & $5891 \cdot 53.2 .2$ & - & $\cdot$ & 15.05 & . & . \\
\hline & $6095-(8.7-7$ & : & . & $\begin{array}{l}3.89 \\
9.66\end{array}$ & 2.56 & 5.87 \\
\hline & 18879799.0 & . & . & 9.0 & : & 96 \\
\hline & $20556-13-2$ & . & . & 11.59 & . & 7.62 \\
\hline & 24305:27.9 & . & . & . & . & 11.04 \\
\hline & 30670.30 .5 & . & . & . & . & 7.45 \\
\hline & 42406-77.99 & . & . & . & . & . \\
\hline & 203303-01.9 & . & . & 2.80 & . & 12.08 \\
\hline & $45234+22-4$ & . & . & . & . & . \\
\hline
\end{tabular}

Figure 6. Binding affinities of the ligands to the SlitOBPI mutants. (A) Binding affinities of mutant Thr9. (B) Binding affinities of mutant Glu98. (C) Binding affinities of mutant Trp37. Final solutions of $2 \mu \mathrm{M}$ SlitOBP1 protein and $2 \mu \mathrm{M} 1-\mathrm{NPN}$ were titrated with $1 \mathrm{mM}$ solution of each ligand in methanol to final concentrations of $0-20 \mu \mathrm{M}$. The figure reported averages of three replicates. (D) The Ki values of the binding affinities. Values are means of three independent experiments. Ligands concentration $>20 \mu M$ for half-maximal relative fluorescence intensity was represented as “-”. 
Besides its broad binding spectrum, in particular, this class of proteins has another advantage that they can be easily modified by genetic engineering techniques to improve their binding specificities [49]. The results of the molecular simulation suggested that hydrogen bonds and electrostatic interaction played important roles in increasing the structural stability of the protein-ligand complexes. Oxygen atoms of many ligands, including 45234-02-4, 678-39-7, 754-96-1, could form hydrogen bond with the Thr9, Ser56, and Trp37, which lay in the middle of the binding pocket of the protein. The conjugative effect also plays an important role, for example, in the binding with chemical 45234-02-4, 24305-27-9, 2545-89-3 and 20556-13-2. The surrounding residues, which were close to the interactions, were identified as key residues in the binding activities. After nine key amino acids were mutated to alanine, respectively, the binding activities of these mutants to the screened compounds were evaluated. The evaluations were important in assessing the matching degree between the ligand and SlitOBP1 in orientation and conformation, as well as in comparing the binding modes of different ligands to the protein [50]. In this study, four scoring functions were applied to improve the result by reaching the assessment consistency. After mutated these key residues, the mean value of the function scores showed that most of the docking results were lower than the wild-type complexes (Table 3). Moreover, the mutant of both Phe12 and Phe118 resulted in lower docking scores to all the tested ligands. The results suggested these amino acids could regulate the interactions between the protein and the ligands. Thr9, Trp37, Ser56, Glu98 and Arg110 seemed to be indispensable to the formation of hydrogen bonds and electrostatic interaction. The phenomenon was also observed in previous reports; for instance, Ser56 of BmorPBP [51], which was also identified as key amino acid at the binding entrance, and can influence the specific pheromone binding. In addition, the Ser52 and Thr57 of LUSH were found to be able to determine the binding specificity of LUSH by forming hydrogen bonds with hydroxyl of alcohol [52]. Moreover, a study proved that the Phe 89 could control the access of a ligand inside the binding cavity of the protein [53]. Taking together, the results showed that these amino acids at the entrance or middle of the binding cavity are critical for determining the binding specificity of the protein. However, some of the docking results after these mutants showed better scores compared with previous ones. This may be due to the rearrangement and re-conformation of the binding cavity to form new binding site to the ligands with formation of non-covalent forces. And to provide practical verification, the competitive binding assays were carried out. None of the chemicals could compete $50 \% 1-\mathrm{NPN}$, even when the ligand concentration reached $20 \mu \mathrm{M}$ or higher after mutating the Phe12, Ile52. A possible and direct explanation is that the ligands could not be recognized by the mutant and could not enter in the binding cavity due to the loss of hydrogen bonding. The mutation of Thr9, Glu98 and Trp37 showed lower affinities towards most of the ligands compared with the wild type, which was consistent with the result of computer simulation. Perhaps because of the capacity of the mutant to establish new van der Waals interactions or other force, mutant Thr9, Glu98 and Trp37 showed a slight increase in binding to some ligands. Some results showed inconsistency with the computer simulation, such as the binding activity of the mutant Thr9 to 203303-01-9 and the mutant Glu98 to 50-24-8, this may be due to the complex binding mechanism or different binding mode of long chain chemicals, which needs further investigation. Overall, the selectivity and specificity of SlitOBP can be further increased by targeted mutagenesis.

OBPs possess many desirable features such as broad binding affinities to different structure compounds and stable at different $\mathrm{pH}$ values, making them suitable for biosensors [54]. The fact that the OBP is able to interact with a broad range of compounds could be effectively used in an olfactory element-based biosensor [55,56]. In this study, we investigated the binding capabilities of SlitOBP1 using a combination of computer simulation methods, mutagenesis, and competitive binding assay. As a result, SlitOBP1 showed specificities towards diverse organic compounds, which is promising for building a multisensor device for various ligands discrimination. Future work will focus on the strategy to adopt for transducing chemical information encoded in the molecule into electrical signals that could be easily measured, amplified and processed. Moreover, the exploration method of the SlitOBP1 in this study could provide a platform and role model to explore other elements in the olfactory systems as biosensor or other applications.

\section{Supplementary Material}

Supplementary Figure 1.

http://www.ijbs.com/v11p0075s1.pdf

\section{Acknowledgements}

The work supported by the National Natural Science Foundation of China for funding this work under grant no. 31071713.

\section{Competing Interests}

The authors have declared that no competing 


\section{interest exists.}

\section{References}

[1] Demirev PA, Feldman AB, Kowalski P, et al. Top-down proteomics for rapid identification of intact microorganisms. Anal chem 2005;77:7455-61.

[2] Varriale A, Staiano M, Marzullo VM, et al. A surface plasmon resonance-based biochip to reveal traces of ephedrine. Analytical Methods 2012;4:1940-4.

[3] Pellejero I, Agustí J, Urbiztondo M, et al. Nanoporous silicalite-only cantilevers as micromechanical sensors: Fabrication, resonance response and VOCs sensing performance. Sensors and Actuators B: Chemical 2012;168:74-82.

[4] Sankaran S, Panigrahi S, Mallik S. Odorant binding protein based biomimetic sensors for detection of alcohols associated with Salmonella contamination in packaged beef. Biosensors and Bioelectronics 2011;26:3103-9.

[5] Lee SH, Ko HJ, Park TH. Real-time monitoring of odorant-induced cellular reactions using surface plasmon resonance. Biosensors and Bioelectronics 2009;25:55-60.

[6] Vidic JM, Grosclaude J, Persuy M-A, et al. Quantitative assessment of olfactory receptors activity in immobilized nanosomes: a novel concept for bioelectronic nose. Lab on a Chip 2006;6:1026-32.

[7] Lee SH, Jin HJ, Song HS, et al. Bioelectronic nose with high sensitivity and selectivity using chemically functionalized carbon nanotube combined with human olfactory receptor. Journal of biotechnology 2012;157:467-72.

[8] Jin HJ, Lee SH, Kim TH, et al. Nanovesicle-based bioelectronic nose platform mimicking human olfactory signal transduction. Biosensors and Bioelectronics 2012;35:335-41.

[9] Wei Y, Brandazza A, Pelosi P. Binding of polycyclic aromatic hydrocarbons to mutants of odorant-binding protein: a first step towards biosensors for environmental monitoring. Biochimica et Biophysica Acta (BBA)-Proteins and Proteomics 2008;1784:666-71.

[10] Vogt RG, Riddiford LM, Prestwich GD. Kinetic properties of a sex pheromone-degrading enzyme: the sensillar esterase of Antheraea polyphemus. Proceedings of the National Academy of Sciences 1985;82:8827-31.

[11] Pelosi P, Zhou J-J, Ban L, et al. Soluble proteins in insect chemical communication. Cellular and Molecular Life Sciences CMLS 2006;63:1658-76.

[12] Lu Y, Li H, Zhuang S, et al. Olfactory biosensor using odorant-binding proteins from honeybee: Ligands of floral odors and pheromones detection by electrochemical impedance. Sensors and Actuators B: Chemical 2014;193:420-7.

[13] Di Pietrantonio F, Cannatà D, Benetti M, et al. Detection of odorant molecules via surface acoustic wave biosensor array based on odorant-binding proteins. Biosensors and Bioelectronics 2013;41:328-34.

[14] Di Pietrantonio F, Benetti M, Dinca V, et al. Tailoring odorant-binding protein coatings characteristics for surface acoustic wave biosensor development. Applied Surface Science 2013;302:250-255.

[15] Persaud KC. Biomimetic Olfactory Sensors. Sensors Journal, IEEE 2012;12:3108-12.

[16] Göpel W, Ziegler C, Breer H, et al. Bioelectronic noses: a status report part I. Biosensors and Bioelectronics 1998;13:479-93.

[17] Silva C, Matamá T, Azoia NG, et al. Odorant binding proteins: a biotechnological tool for odour control. Applied microbiology and biotechnology 2013; 98:1-10.

[18] Pelosi P. Physiological and artificial biosensor for odour recognition systems. Molecular Electronics: Bio-sensors and Bio-computers. Springer. 2003: 379-88.

[19] Pelosi P, Mastrogiacomo R, Iovinella I, et al. Structure and biotechnological applications of odorant-binding proteins. Applied microbiology and biotechnology 2014;98:61-70

[20] Leal WS. Odorant reception in insects: roles of receptors, binding proteins, and degrading enzymes. Annual review of entomology 2013;58:373-91.

[21] Forstner M, Breer H, Krieger J. A receptor and binding protein interplay in the detection of a distinct pheromone component in the silkmoth Antheraea polyphemus. Int J Biol Sci 2009;5:745-57.

[22] Fan J, Francis F, Liu Y, et al. An overview of odorant-binding protein functions in insect peripheral olfactory reception. Genet Mol Res 2011;10:3056-69.

[23] Zhong T, Yin J, Deng S, et al. Fluorescence competition assay for the assessment of green leaf volatiles and trans- $\beta$-farnesene bound to three odorant-binding proteins in the wheat aphid Sitobion avenae (Fabricius). Journal of insect physiology 2012;58:771-81.

[24]. Vidic J. Bioelectronic noses based on olfactory receptors: Intelligent and Biosensor, Vernon S. Somerset; 2010: 67-69.

[25] ZHONG GH, Li MM, Hu MY, et al. Cloning and Sequence Analysis of SlitGOBP1 Encoding a General Odorant Binding Protein 1 from Spodoptera litura. Journal of South China Agricultural University 2008;2:38-43

[26] Qi C, Jin L, Hong P. A simple artificial diet for mass rearing of some noctuid species. Entomological knowledge 2000;37:8-10.

[27] Šali A, Blundell TL. Comparative protein modelling by satisfaction of spatial restraints. Journal of molecular biology 1993;234:779-815.

[28] Jiang Q-Y, Wang W-X, Zhang Z, et al. Binding specificity of locust odorant binding protein and its key binding site for initial recognition of alcohols. Insect biochemistry and molecular biology 2009;39:440-7.

[29] Liithy R, Bowie JU, Eisenberg D. Assessment of protein models with three-dimensional profiles. Nature 1992;356:83-5.

[30] Ramachandran G, Ramakrishnan Ct, Sasisekharan V. Stereochemistry of polypeptide chain configurations. Journal of molecular biology 1963;7:95-9.
[31] Wu G, Robertson DH, Brooks CL, et al. Detailed analysis of grid-based molecular docking: A case study of CDOCKER-A CHARMm-based MD docking algorithm. Journal of computational chemistry 2003;24:1549-62.

[32] Bradford MM. A rapid and sensitive method for the quantitation of microgram quantities of protein utilizing the principle of protein-dye binding. Analytical biochemistry 1976;72:248-54.

[33] Yi X, Wang P, Wang Z, et al. Involvement of a Specific Chemosensory Protein from Bactrocera dorsalis in Perceiving Host Plant Volatiles. Journal of chemical ecology 2014; 40:1-9.

[34] Nguyen JT, Turck CW, Cohen FE, et al. Exploiting the basis of proline recognition by $\mathrm{SH} 3$ and WW domains: design of $\mathrm{N}$-substituted inhibitors. Science 1998;282:2088-92.

[35] Jorgensen WL, Chandrasekhar J, Madura JD, et al. Comparison of simple potential functions for simulating liquid water. The Journal of chemical physics 1983;79:926-35.

[36] Liu Q, Wang H, Li H, et al. Impedance sensing and molecular modeling of an olfactory biosensor based on chemosensory proteins of honeybee. Biosensors and Bioelectronics 2013;40:174-9.

[37] Ramoni R, Staiano M, Bellucci S, et al. Carbon nanotube-based biosensors. Journal of Physics: Condensed Matter 2008;20:474201.

[38] Glatz R, Bailey-Hill K. Mimicking nature's noses: from receptor deorphaning to olfactory biosensing. Progress in neurobiology 2011;93:270-96.

[39] Damberger F, Horst R, Wüthrich K, et al. NMR characterization of a $\mathrm{pH}$-dependent equilibrium between two folded solution conformations of the pheromone-binding protein from Bombyx mori. Protein Science 2000;9:1038-41.

[40] Thomsen R, Christensen MH. MolDock: a new technique for high-accuracy molecular docking. Journal of medicinal chemistry 2006;49:3315-21.

[41] Alfinito E, Pennetta C, Reggiani L. A network model to correlate conformational change and the impedance spectrum of single proteins. Nanotechnology 2008;19:065202.

[42] Cheng T, Li X, Li Y, et al. Comparative assessment of scoring functions on a diverse test set. Journal of chemical information and modeling 2009;49:1079-93.

[43] Liu NY, Liu CC, Dong SL. Functional differentiation of pheromone-binding proteins in the common cutworm Spodoptera litura. Comparative Biochemistry and Physiology Part A: Molecular \& Integrative Physiology 2013;165:254-62.

[44] Liu NY, He P, Dong SL. Binding properties of pheromone-binding protein 1 from the common cutworm Spodoptera litura. Comparative Biochemistry and Physiology Part B: Biochemistry and Molecular Biology 2012;161:295-302.

[45] Jahnke A, Huber S, Temme C, et al. Development and application of a simplified sampling method for volatile polyfluorinated alkyl substances in indoor and environmental air. Journal of Chromatography A 2007:1164:1-9.

[46] Fontell K, Lindman B. Fluorocarbon surfactants. Phase equilibriums and phase structures in aqueous systems of a totally fluorinated fatty acid and some of its salts. The Journal of Physical Chemistry 1983;87:3289-97.

[47] Johnston G, Curtis D, Davies J, et al. Spinal interneurone excitation by conformationally restricted analogues of L-glutamic acid. Nature 1974;248:804-805.

[48] Tannock IF, de Wit R, Berry WR, et al. Docetaxel plus prednisone or mitoxantrone plus prednisone for advanced prostate cancer. New England Journal of Medicine 2004;351:1502-12.

[49] Ban L, Zhang L, Yan Y, et al. Binding properties of a locust's chemosensory protein. Biochemical and biophysical research communications 2002;293:50-4.

[50] Ban L, Scaloni A, Brandazza A, et al. Chemosensory proteins of Locusta migratoria. Insect molecular biology 2003;12:125-34.

[51] Ramoni R, Bellucci S, Grycznyski I, et al. The protein scaffold of the lipocalin odorant-binding protein is suitable for the design of new biosensors for the detection of explosive components. Journal of Physics: Condensed Matter 2007;19:395012.

[52] Shoichet BK, Kuntz ID, Bodian DL. Molecular docking using shape descriptors. Journal of Computational Chemistry 1992;13:380-97.

[53] Sandler BH, Nikonova L, Leal WS, et al. Sexual attraction in the silkworm moth: structure of the pheromone-binding-protein-bombykol complex. Chemistry \& biology 2000;7:143-51.

[54] Thode AB, Kruse SW, Nix JC, et al. The role of multiple hydrogen-bonding groups in specific alcohol binding sites in proteins: insights from structural studies of LUSH. Journal of molecular biology 2008;376:1360-76.

[55] Bianchet MA, Bains G, Pelosi P, et al. The three-dimensional structure of bovine odorant binding protein and its mechanism of odor recognition. Nature Structural \& Molecular Biology 1996;3:934-9.

[56] Sun M, Liu Y, Wang G. Expression patterns and binding properties of three pheromone binding proteins in the diamondback moth, Plutella xyllotella. Journal of insect physiology 2013;59:46-55.

[57] Pevsner J, Hou V, Snowman A, et al. Odorant-binding protein. Characterization of ligand binding. Journal of Biological Chemistry 1990;265:6118-25.

[58] Ko HJ, Park TH. Enhancement of odorant detection sensitivity by the expression of odorant-binding protein. Biosensors and Bioelectronics 2008;23:1017-23 